\title{
Factors Associated with Attempted External Cephalic Version for Fetal Malpresentation at Term
}

\author{
Meghana Limaye, MD ${ }^{1}$ Najma Abdullahi, MPH ${ }^{2}$ Phinnara Has, MS² Valery A. Danilack, MPH, PhD ${ }^{2}$ \\ Rosemary Froehlich, MD ${ }^{3}$ Erika Werner, MD, MS²
}

${ }^{1}$ Department of Obstetrics \& Gynecology, NYU Langone Medical Center, New York, New York

2 Department of Obstetrics \& Gynecology, Warren Alpert Medical School of Brown University, Women \& Infants Hospital of Rhode Island, Providence, Rhode Island

${ }^{3}$ Department of Obstetrics, Gynecology, and Reproductive Sciences, University of Pittsburgh Medical Center, Pittsburgh, Pennsylvania

Address for correspondence Meghana Limaye, MD, Department of Obstetrics \& Gynecology, NYU Langone Medical Center, 462 1st Ave, New York, NY 10016 (e-mail: Meghana.limaye@gmail.com).

Am J Perinatol Rep 2019;9:e323-e327.

\begin{abstract}
Keywords

- external cephalic version

- fetal malpresentation

- prevention of primary cesarean delivery

- utilization of external cephalic version

Objective To assess differences in patient characteristics between women who did and did not undergo attempted external cephalic version (ECV) for fetal malpresentation at term.

Study Design This was a retrospective cohort study of women with a singleton gestation and noncephalic presentation at $>37.0$ weeks between October 2014 and October 2015. We compared demographic and clinical characteristics of women who did and did not undergo attempted ECV and assessed the reasons that women did not attempt ECV.

Results Among 215 women, only 51 (24\%) attempted ECV. There were no differences in age, race, insurance type, or body mass index between women who underwent attempted ECV and those who did not. Women who underwent ECV were significantly more likely to have had a prior vaginal delivery ( 69 vs. $36 \%, p<0.001$ ). Seventy-six women (46\%) declined ECV. Women who declined ECV were more likely to be nulliparous than those who accepted the procedure (66 vs. $29 \%, p<0.001)$. Among women who had ECV, the success rate was $55 \%$. There were no adverse events after attempted ECV in this cohort.

Conclusion Among women with fetal malpresentation at term, those without a prior vaginal delivery were significantly less likely to undergo attempted ECV.
\end{abstract}

In the United States today, nearly one in three women give birth by cesarean delivery. ${ }^{1}$ Over the past 15 years, there has been a rapid increase in the cesarean delivery rate without evidence of corresponding decreases in maternal or neonatal morbidities or mortality, suggesting that cesarean delivery may be overused. ${ }^{2}$ Because repeat cesarean delivery has an increased risk of severe maternal morbidity and women with one cesarean delivery have a high likelihood of repeat cesarean delivery, there has been a recent focus on safely

received

April 12, 2019 accepted after revision June 11, 2019
DOI https://doi.org/

10.1055/s-0039-1695748. ISSN 2157-6998. decreasing the rate of primary cesarean delivery with the goal of ultimately decreasing the rate of repeat cesarean delivery. In 2016, the primary cesarean delivery rate in the United States was $21.8 \%^{3}$ Breech presentation, though it occurs in only $3.8 \%$ of all term pregnancies, ${ }^{4}$ accounted for $17 \%$ of these primary cesarean deliveries. ${ }^{2}$

External cephalic version (ECV) offers a potential means to decrease the need for cesarean delivery due to breech presentation by converting the fetus to cephalic presentation. It is a
Copyright $\odot 2019$ by Thieme Medical Publishers, Inc., 333 Seventh Avenue, New York, NY 10001, USA. Tel: +1(212) 584-4662.
License terms

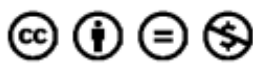


safe procedure with no demonstrated increase in perinatal morbidity or mortality, ${ }^{5}$ and a pooled success rate of $58 \%$ based on data from meta-analysis. ${ }^{6}$ Success rates have been shown to increase with the use of terbutaline and regional anesthesia., 7,8 Additionally, ECV has been demonstrated to be cost-effective as long as the success rate of the procedure is $>32 \% .{ }^{9}$ Given the low risk of ECV to mother and fetus and the increased likelihood of vaginal delivery after successful ECV, the American College of Obstetrics and Gynecology (ACOG) published guidelines in February 2016 which recommended that providers offer ECV with tocolysis to all eligible patients. ${ }^{4}$ Internationally, ECV is offered to as many as 70 to $80 \%$ of eligible mothers, ${ }^{10,11}$ but there is little comparable data evaluating US practice patterns.

Given this lack of data on ECV practices in the United States, we sought to determine the rate of ECV uptake at one large tertiary care hospital and to determine the differences in patient characteristics between women who did and did not undergo attempted ECV. In particular, ECV success rates are reported to be lower in nulliparous women, ${ }^{10,12}$ but there is little data available regarding the uptake of the procedure in this group. We hypothesized that because of the lower reported success rates of ECV, women without a prior vaginal delivery would be less likely to undergo an attempted ECV. If true, this could represent an opportunity to improve uptake of ECV among nulliparous women with malpresentation in the hopes of reducing the primary cesarean delivery rate.

\section{Materials and Methods}

We conducted a retrospective cohort study of women at term ( $\geq 37$ weeks) with a singleton gestation and fetal malpresentation, who underwent attempted ECV or cesarean delivery for malpresentation at one tertiary care hospital between October 2014 and October 2015. The study population of all women at term with a singleton gestation and fetal malpresentation was identified using International Classification of Diseases, ninth edition (ICD9) codes for malpresentation during the delivery hospitalization and the Current Procedures Terminology (CPT) code for ECV during any emergency department visit (ICD9 652.0-652.9, CPT 59412). Women were excluded if they had a multifetal gestation, more than one prior cesarean delivery, any contraindication to vaginal delivery (placenta previa, history of classical cesarean delivery, or myomectomy entering the uterine cavity), or whose third trimester prenatal records were unavailable for review. All charts were screened for exclusion criteria and reasons for exclusion were recorded.

The study was approved by the Institutional Review Board at Women \& Infant's Hospital, Providence RI. Women \& Infants is a large tertiary care hospital with 8,000 deliveries per year. At our institution, women with fetal malpresentation are typically scheduled for ECV in the 37th week, which is performed as an outpatient procedure in the emergency department. Attempted ECV is followed by an hour of continuous fetal monitoring and if reassuring the patient is discharged home. If the ECV is unsuccessful, based on patient preference women are either scheduled for cesarean delivery or offered a second ECV in the 39th week with the addition of neuraxial analgesia.

Two authors (ML, NA) performed detailed data abstraction by individual electronic chart review. Data abstracted included maternal demographic information and medical comorbidities, prenatal care provider, documentation of malpresentation between 32 and 36 weeks, whether ECV was offered and performed, details of ECV, and delivery information. If ECV was not performed, the reason ECV was not performed was also recorded based on provider documentation in the electronic medical record. Neonatal Apgar score, birth weight, and cord gases were recorded if available. Following complete data abstraction, $10 \%$ of the charts were randomly selected and abstracted a second time to ensure internal data validity. Any discrepancies noted in reabstraction were corrected after reviewing the patient chart. For eight variables where $>25 \%$ of the values had inconsistencies, a single reviewer (ML) reabstracted all charts.

The primary outcome was defined as the percentage of women who underwent attempted ECV. We hypothesized that women with a history of prior vaginal delivery were more likely to have an attempted ECV than women without a prior vaginal delivery and an a priori power calculation was performed for this hypothesis. Assuming a 30\% overall cesarean delivery rate and that $40 \%$ of women are nulliparous, we estimated that $58 \%$ of our population would have had no prior vaginal delivery. We estimated that $50 \%$ of women with a prior vaginal delivery would have an attempted ECV. With a power of $80 \%$ and a type 1 error of $5 \%$, we estimated that a sample size of 192 would be needed to detect a difference of $20 \%$ in the rate of ECV performed for women without a prior vaginal delivery.

Baseline characteristics were determined for the entire cohort. Women who had an attempted ECV were compared with women who did not undergo ECV. Categorical variables were compared using chi-squared or Fisher's exact test, and continuous variables were compared using Student's $t$-test and nonparametric Wilcoxon rank-sum test. A $p$-value $<0.05$ was used to define statistical significance. All statistical analyses were performed using Stata 13.1 (College Station, TX).

\section{Results}

We identified 644 women with our specified ICD9 and CPT codes who delivered between October 2014 and October 2015; 215 women met study criteria and were included for analysis (-Fig. 1). Only 51 of these 215 women (24\%) underwent attempted ECV. When women who had attempted ECV were compared with those who did not, there were no differences in age, race, insurance type, or body mass index (-Table 1). Women who had an attempted ECV were significantly more likely to have experienced a prior vaginal delivery than women who did not undergo the procedure (69 vs. $36 \%, p<0.001$ ). Women who underwent attempted ECV were also more likely to have a posterior placenta and less likely to have had a prior cesarean delivery (-Table 1). 


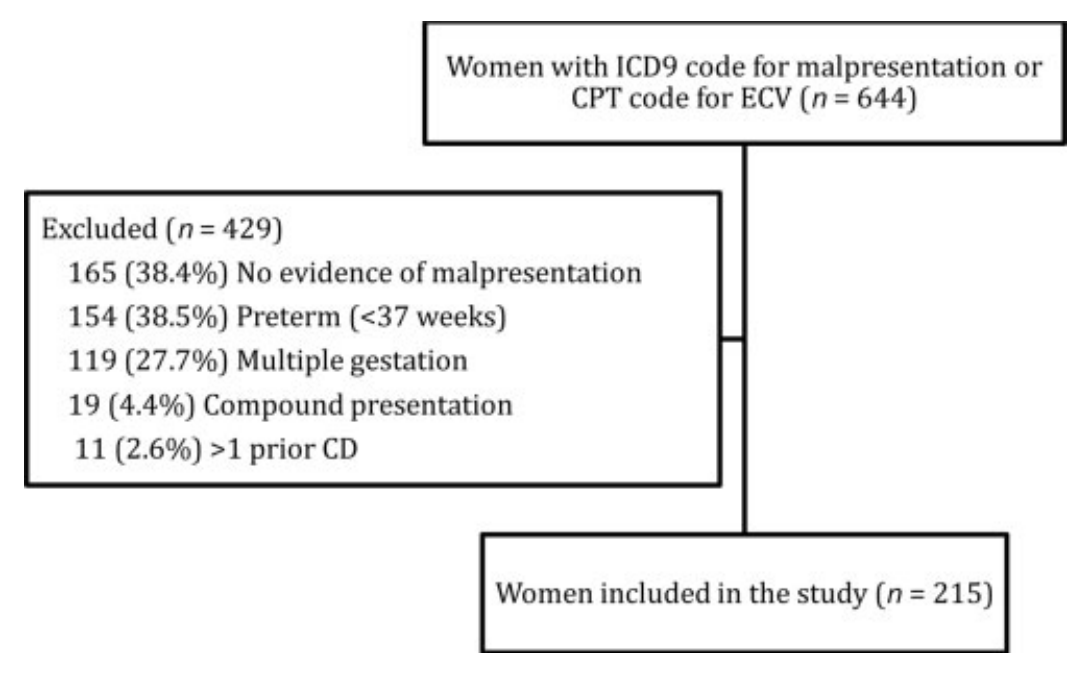

Fig. 1 Study design. CD, cesarean delivery; CPT, Current Procedures Terminology; ECV, external cephalic version.

Of the 164 women (76\%) who did not undergo ECV but had a malpresenting fetus at term, 76 (46.3\%) women were offered but declined an ECV per documentation in the medical record. Women who declined ECV were more likely to be nulliparous than those who accepted the procedure (66 vs. $29 \%, p<0.001)$. Twenty-nine women (17\%) lacked documentation of an offer of attempted ECV in their prenatal medical record. An additional 23 women (14\%) were not diagnosed with fetal malpresentation until they presented in labor or after spontaneous rupture of membranes. Other reasons that ECV was not attempted are listed (-Table 2 ). Of the women who did not have an attempted ECV, only $70 \%$ had documentation of fetal presentation between 32 and 36 weeks' gestation, compared with $90 \%$ of women who underwent attempted ECV.

The overall success rate of ECV in this study was 55\% (28/51). Prior to the attempted procedure, 47\% (24/51) of women received terbutaline, $14 \%$ (7/51) received intravenous pain medication, and $6 \%$ (3/51) of women had regional anesthesia. There were no complications or emergent cesarean deliveries attributed to attempted ECV. A total of $42 \%$ (22/51) of women who had ECVs went on to deliver vaginally, but only $7 \%(11 / 164)$ of women who did not undergo ECV were able to achieve a vaginal delivery, due to spontaneous version before delivery.

\section{Discussion}

ACOG guidelines suggest that all patients with fetal malpresentation at term should be offered ECV. ${ }^{4}$ If most eligible patients underwent attempted ECV, this would contribute to decreasing the primary cesarean delivery rate. However, in our study only $24 \%$ of eligible patients actually underwent attempted ECV, and women without a prior vaginal delivery were significantly less likely to have an attempted ECV. Our data are consistent with a recent study by Son et al, who noted that only $31 \%$ of eligible women in a US population actually had an attempted ECV, and nulliparous women were significantly less likely to undergo the procedure. ${ }^{5}$

This study adds to the existing literature by analyzing the reasons that ECV was not attempted. The most common reason was that women declined the procedure. It is unclear why so many women decline ECV in the United States, when high rates of acceptance of ECV (>90\%) have been reported in several other countries. ${ }^{10,11,13}$

Some small qualitative studies in Europe ${ }^{14,15}$ have suggested that women may not accept ECV due to fear of pain during the procedure, perceived low likelihood of ECV success, and possibility of fetal distress, but this has not been shown in the United States.

It is also possible that providers counsel women against ECV if they think the procedure will be unsuccessful, such as in a nulliparous woman. Due to the limitations of the medical record and the retrospective nature of the study, we were not able to determine why women declined ECV in this population.

In our study, the success rate of ECV in women without a prior vaginal delivery was $25 \%$ (4/16), consistent with the rate documented in the literature, ${ }^{10,12}$ and nulliparous women were less likely to have an attempted ECV. This does suggest either a patient or provider bias toward not attempting ECV in these nulliparous women who have a lower rate of successful ECV and are also most at risk of cesarean delivery. However, details were not available in the medical record about the counseling patients received.

Additionally, our study identified that nearly $20 \%$ of women who were eligible for ECV had no documentation that the procedure was offered, representing a missed opportunity for intervention and prevention of a primary cesarean delivery. Twenty-six percent of all patients with malpresentation during their delivery hospitalization had no documentation of fetal presentation in the third trimester and these women were far more likely to miss the opportunity for ECV.

This study had several limitations. It was performed at a single institution and reflected practices particular to that center, thus generalizability of the findings may be limited. The ACOG practice bulletin recommending documentation of presentation and offering ECV to all eligible patients was published in February 2016, after the time period of this study. It is possible that prior to this recommendation, providers may have offered ECV less frequently. The retrospective nature of our study, reliant on chart abstraction, is 
Table 1 Characteristics of women who underwent attempted ECV compared with women who did not

\begin{tabular}{|c|c|c|c|}
\hline Characteristics & $\begin{array}{l}\text { Attempted } \\
\mathrm{ECV}(n=51)\end{array}$ & $\begin{array}{l}\text { No attempted } \\
\text { ECV }(n=164)\end{array}$ & $p$-Value \\
\hline Age at delivery (SD) & $32.7(5.2)$ & $31.0(5.9)$ & $0.11^{\mathrm{a}}$ \\
\hline $\begin{array}{l}\text { Prenatal care } \\
\text { provider }(n, \%)\end{array}$ & & & \multirow[t]{3}{*}{$0.09^{b}$} \\
\hline Academic & $8(15.7)$ & $11(6.7)$ & \\
\hline Private group & $43(84.3)$ & $153(93.3)$ & \\
\hline \multicolumn{3}{|l|}{ Race $(n, \%)$} & \multirow[t]{7}{*}{$0.26^{\mathrm{b}}$} \\
\hline White & $32(62.8)$ & $100(63.4)$ & \\
\hline Black & $0(-)$ & $6(3.7)$ & \\
\hline Hispanic & $8(15.7)$ & $32(19.5)$ & \\
\hline Asian & $6(11.8)$ & $7(4.3)$ & \\
\hline Other & $3(5.9)$ & $12(7.3)$ & \\
\hline Unknown & $2(3.9)$ & $3(1.8)$ & \\
\hline \multicolumn{3}{|l|}{ Insurance type $(n, \%)$} & \multirow[t]{4}{*}{$0.93^{\mathrm{b}}$} \\
\hline Uninsured & $0(-)$ & $2(1.2)$ & \\
\hline Medicaid & $22(43.1)$ & $67(40.9)$ & \\
\hline Commercial & 29 (56.9) & $95(57.9)$ & \\
\hline $\begin{array}{l}\begin{array}{l}\text { Prior vaginal } \\
\text { delivery }(n, \%)\end{array} \\
\end{array}$ & $35(68.6)$ & 59 (35.9) & $<0.001^{b}$ \\
\hline Nulliparous ( $n, \%)$ & $16(31.4)$ & $85(51.8)$ & $0.02^{\mathrm{b}}$ \\
\hline $\begin{array}{l}\text { Prior cesarean } \\
\text { delivery }(n, \%)\end{array}$ & $2(3.9)$ & $27(16.5)$ & $0.02^{\mathrm{b}}$ \\
\hline \multicolumn{3}{|l|}{ Placental location $(n, \%)$} & \multirow[t]{6}{*}{$0.02^{b}$} \\
\hline Fundal & $3(5.9)$ & $15(9.2)$ & \\
\hline Anterior & $19(37.3)$ & $70(42.7)$ & \\
\hline Posterior & $26(50.9)$ & $45(27.4)$ & \\
\hline Lateral & $1(1.9)$ & $9(5.5)$ & \\
\hline Unknown & $2(3.9)$ & $25(15.2)$ & \\
\hline \multicolumn{3}{|l|}{$\begin{array}{l}\text { Pre-pregnancy } \\
\text { BMI }\left(\mathrm{kg} / \mathrm{m}^{2}\right)\end{array}$} & \multirow[t]{5}{*}{$0.49^{b}$} \\
\hline$<24.9$ & $29(58.0)$ & $76(47.5)$ & \\
\hline $25.0-29.9$ & $13(26.0)$ & $43(26.9)$ & \\
\hline $30.0-39.9$ & $7(14.0)$ & $31(19.4)$ & \\
\hline$\geq 40.0$ & $1(2.0)$ & $10(6.3)$ & \\
\hline $\begin{array}{l}\text { Gestational weight } \\
\text { gain (mean in } \mathrm{kg}, \mathrm{SD} \text { ) }\end{array}$ & $13.5(6.4)$ & $13.4(6.1)$ & $0.87^{\mathrm{a}}$ \\
\hline \multicolumn{4}{|l|}{ Medical comorbidities } \\
\hline Pre-existing diabetes & $1(1.9)$ & $2(1.2)$ & $.562^{\mathrm{b}}$ \\
\hline Gestational diabetes & $5(9.8)$ & $15(9.2)$ & $1.00^{\mathrm{b}}$ \\
\hline $\begin{array}{c}\text { Pre-existing } \\
\text { hypertension }\end{array}$ & $1(1.9)$ & $9(5.5)$ & $0.462^{b}$ \\
\hline $\begin{array}{l}\text { Pregnancy induced } \\
\text { hypertension }\end{array}$ & $7(13.7)$ & $8(4.9)$ & $0.05^{\mathrm{b}}$ \\
\hline $\begin{array}{l}\text { Estimated fetal weight } \\
>90 \text { th percentile }\end{array}$ & $2(6.9)$ & $8(8.6)$ & $1.00^{\mathrm{b}}$ \\
\hline $\begin{array}{l}\text { Birth weight } \\
\text { (mean in grams, SD) }\end{array}$ & $3363(527)$ & 3302 (459) & $0.46^{c}$ \\
\hline
\end{tabular}

Abbreviations: BMI, body mass index; ECV, external cephalic version; SD, standard deviation.

Note: Data presented as $n(\%)$ unless otherwise noted.

${ }^{a}$ Compared with Wilcoxon rank-sum test.

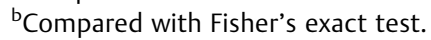

'Compared with Student's $t$-test.
Table 2 Reasons cited in the medical record for not performing ECV in cases of malpresentation $(n=164)$

\begin{tabular}{|l|l|}
\hline & $n(\%)$ \\
\hline Patient declined ECV & $76(46.3)$ \\
\hline Malpresentation diagnosed while in labor & $19(11.6)$ \\
\hline Planned repeat CD & $18(10.9)$ \\
\hline ECV planned but spontaneous version occurred & $11(6.7)$ \\
\hline $\begin{array}{l}\text { Malpresentation diagnosed after spontaneous } \\
\text { rupture of membranes }\end{array}$ & $4(2.4)$ \\
\hline Oligohydramnios at time of planned ECV & $3(1.8)$ \\
\hline Planned elective primary CD & $2(1.2)$ \\
\hline Rupture of membranes prior to planned ECV & $1(0.6)$ \\
\hline Not offered due to suspected fetal macrosomia & $1(0.6)$ \\
\hline No documented discussion of ECV & $29(17.7)$ \\
\hline
\end{tabular}

Abbreviations: ECV, external cephalic version; CD, cesarean delivery.

also a limitation; it is possible that counseling occurred that was not documented in the medical record. In particular, our prenatal charts do not have a uniform area to document counseling on ECV or patient acceptance or refusal of the procedure. Finally, there may be a selection bias inherent in how the study cohort was chosen. While we attempted to get a complete sample of all patients with malpresentation and all patients with an ECV procedure, we may have missed a group of patients with breech presentation in the third trimester who had accepted ECV but had spontaneous version prior to attempted ECV or delivery. Had we been able to capture them, this group would have been included in the "no attempted ECV" group and may have been more likely to have a prior vaginal delivery. Because they were not included, we may have seen a larger difference between the groups in rate of attempted ECV than is truly present.

Our study also has several strengths. While the cohort was generated from one institution, this institution performs roughly $80 \%$ of the deliveries in Rhode Island and includes a wide variety of practice types, including patients cared for by midwives, general practitioners, residents, and Maternal Fetal Medicine physicians. We were also able to obtain a comprehensive list of ECVs attempted at our institution from emergency department billing records. Additionally, this study explores an under-investigated topic: the utilization of ECV in eligible patients.

In conclusion, we found that women who have not had a prior vaginal delivery were significantly less likely to attempt ECV. This is particularly concerning as this population contributes significantly to the primary cesarean delivery rate. This study highlights an opportunity to improve practice patterns so that a higher proportion of eligible women undergo ECV and avoid primary cesarean delivery. Future interventions should focus on understanding the reasons women decline ECV, increasing the uptake of ECV among women without a history of a vaginal delivery through provider and patient education, encouraging universal documentation of fetal presentation for all women in the third trimester, and ensuring that all eligible women are offered ECV. 
Presented at

ACOG Annual Clinical and Scientific Meeting, May 6-9, 2017, San Diego, CA

\section{Conflict of Interest}

None.

\section{References}

1 Martin JA, Hamilton BE, Osterman MJK, Driscoll AK, Drake P. Births: final data for 2016. Natl Vital Stat Rep 2018;67(01):1-55

2 American College of Obstetricians and Gynecologists; Society for Maternal-Fetal Medicine. Obstetric care consensus no. 1: safe prevention of the primary cesarean delivery. Obstet Gynecol 2014;123(03):693-711

3 Hamilton BE, Martin JA, Osterman MJ, Curtin SC, Matthews TJ. Births: final data for 2014. Natl Vital Stat Rep 2015;64(12):1-64

4 ACOG. ACOG Practice Bulletin 161: External Cephalic Version. Obstet Gynecol 2016;127:54-61

5 Son M, Roy A, Grobman WA, Miller ES. Association between attempted external cephalic version and perinatal morbidity and mortality. Obstet Gynecol 2018;132(02):365-370

6 Hofmeyr GJ, Kulier R, West HM. External cephalic version for breech presentation at term. Cochrane Database Syst Rev 2015; (04):CD000083

7 Goetzinger KR, Harper LM, Tuuli MG, Macones GA, Colditz GA. Effect of regional anesthesia on the success rate of external cephalic version: a systematic review and meta-analysis. Obstet Gynecol 2011;118(05):1137-1144

8 Fernandez CO, Bloom SL, Smulian JC, Ananth CV, Wendel GD Jr. A randomized placebo-controlled evaluation of terbutaline for external cephalic version. Obstet Gynecol 1997;90(05):775-779

9 Tan JM, Macario A, Carvalho B, Druzin ML, El-Sayed YY. Costeffectiveness of external cephalic version for term breech presentation. BMC Pregnancy Childbirth 2010;10:3

10 Melo P, Georgiou EX, Hedditch A, Ellaway P, Impey L. External cephalic version at term: a cohort study of 18 years' experience. BJOG 2019;126(04):493-499

11 Vlemmix F, Rosman AN, te Hoven S, et al. Implementation of external cephalic version in the Netherlands: a retrospective cohort study. Birth 2014;41(04):323-329

12 Morgan ER, Hu AE, Brezak AMV, Rowley SS, Littman AJ, Hawes SE. Predictors of a successful external cephalic version: a populationbased study of Washington state births. Women Birth 2019;32 (03):e421-e426

13 Caukwell S, Joels LA, Kyle PM, Mills MS. Women's attitudes towards management of breech presentation at term. J Obstet Gynaecol 2002;22(05):486-488

14 Rosman AN, Vlemmix F, Fleuren MAH, et al. Patients' and professionals' barriers and facilitators to external cephalic version for breech presentation at term, a qualitative analysis in the Netherlands. Midwifery 2014;30(03):324-330

15 Say R, Thomson R, Robson S, Exley C. A qualitative interview study exploring pregnant women's and health professionals' attitudes to external cephalic version. BMC Pregnancy Childbirth 2013;13:4 